\title{
Differential selection on gene translation efficiency between the filamentous fungus Ashbya gossypii and yeasts Huifeng Jiang ${ }^{\dagger 1,2,3}$, Yue Zhang ${ }^{\dagger 2}$, Jun Sun ${ }^{3,4}$, Wen Wang ${ }^{* 2}$ and Zhenglong $\mathrm{Gu}^{* 1}$
}

\begin{abstract}
Address: ${ }^{1}$ Division of Nutritional Sciences, Cornell University, Ithaca, NY 14853, USA, ${ }^{2}$ CAS-Max Planck Junior Research Group, State Key Laboratory of Genetic Resources and Evolution, Kunming Institute of Zoology, Chinese Academy of Sciences (CAS), Kunming, Yunnan 650223, PR China, ${ }^{3}$ Graduate School of Chinese Academy Sciences, Beijing 100039, PR China and ${ }^{4}$ State Key Laboratory of Cellular and Molecular Evolution, Kunming Institute of Zoology, Chinese Academy of Sciences, Kunming 650223, Yunnan Province, PR China

Email: Huifeng Jiang - jianghf3@mail.kiz.ac.cn; Yue Zhang - zhangyue@mail.kiz.ac.cn; Jun Sun - sunj03@mail.kiz.ac.cn; Wen Wang* - wwang@mail.kiz.ac.cn; Zhenglong Gu* - zg27@cornell.edu

* Corresponding authors †Equal contributors
\end{abstract}

Published: 29 December 2008

BMC Evolutionary Biology 2008, 8:343 doi:10.1/86/147/-2/48-8-343
Received: 2 June 2008

Accepted: 29 December 2008

This article is available from: http://www.biomedcentral.com/I47I-2/48/8/343

(C) 2008 Jiang et al; licensee BioMed Central Ltd.

This is an Open Access article distributed under the terms of the Creative Commons Attribution License (http://creativecommons.org/licenses/by/2.0), which permits unrestricted use, distribution, and reproduction in any medium, provided the original work is properly cited.

\begin{abstract}
Background: The filamentous fungus Ashbya gossypii grows into a multicellular mycelium that is distinct from the unicellular morphology of its closely related yeast species. It has been proposed that genes important for cell cycle regulation play central roles for such phenotypic differences. Because A. gossypii shares an almost identical set of cell cycle genes with the typical yeast Saccharomyces cerevisiae, the differences might occur at the level of orthologous gene regulation. Codon usage patterns were compared to identify orthologous genes with different gene regulation between $A$. gossypii and nine closely related yeast species.
\end{abstract}

Results: Here we identified 3,15I orthologous genes between $A$. gossypii and nine yeast species. Two groups of genes with significant differences in codon usage (gene translation efficiency) were identified between A. gossypii and yeasts. 333 genes (Group I) and 552 genes (Group II) have significantly higher translation efficiency in $A$. gossypii and yeasts, respectively. Functional enrichment and pathway analysis show that Group I genes are significantly enriched with cell cycle functions whereas Group II genes are biased toward metabolic functions.

Conclusion: Because translation efficiency of a gene is closely related to its functional importance, the observed functional distributions of orthologous genes with different translation efficiency might account for phenotypic differentiation between $A$. gossypii and yeast species. The results shed light on the mechanisms for pseudohyphal growth in pathogenic yeast species.

\section{Background}

Filamentous and yeast-like growths are two ways for fungi to propagate in nature. Under normal growth conditions, filamentous fungi grow as multicellular mycelium but yeasts form unicellular colonies. Most species in the clade Saccharomycotina are yeasts. In some special environ- ments, these species can switch to pseudohyphal growth that is morphologically similar to filamentous growth, but they can not form real hyphal filaments [1,2]. Ashbya gossypii, however, is classified as a filamentous fungus because it forms natural hyphal filaments for most of its life cycle $[3,4]$. What the nature of the molecular differ- 
ences between A. gossypii and yeasts is that led to such a phenotypic divergence remains an open question. It has been proposed that the divergence might result from a modified cell cycle processes in A. gossypii [4-6]. However, comparative genomics studies reveal that $A$. gossypii shares an almost identical set of cell cycle genes with the typical yeast Saccharomyces cerevisiae [5]. Although loss or gain of genes among different species often play important roles in phenotypic diversity [7], divergence of regulation in orthologous genes can also contribute significantly to this process [8]. The fact that A. gossypii and its closely related yeasts share a very similar set of cell cycle genes prompts us to investigate whether these genes have unique regulation patterns in A. gossypii.

Genetic codes exhibit significant usage bias. Selection for gene translation efficiency is the most common Darwinian explanation for codon usage bias. Under this scenario, codons corresponding to abundant tRNA would be preferentially used [9-12]. Natural selection biases such preferred codons so as to enhance accuracy and speed of protein synthesis in highly expressed genes [13-15]. Therefore, differential translation efficiency reflects protein expression difference, which in return can indicate differential functional requirements for these proteins [14]. Therefore, divergence of translation efficiency could reveal orthologous gene functional differentiation across species [15]. By comparing codon usage patterns for orthologous genes between A. gossypii and nine yeast species [2], we show in this study that in A. gossypii genes with significantly higher translation efficiency are enriched in cell cycle processes, whereas genes with significantly lower translation efficiency are enriched in metabolic processes. Both these findings could account for phenotypic differentiation between multicellular A. gossypii and unicellular yeasts, and would shed light on the evolutionary processes that enable pseudohyphal growth in pathogenic yeast species.

\section{Results}

\section{tRNA adaptation index (tAl)}

Within a genome, codon usage, which matches tRNA pool of the organism, is an important indicator for gene translational efficiency [16]. In order to detect the differences in gene translational efficiency between A. gossypii and yeasts, a gene matrix containing 3,151 orthologous gene groups for A. gossypii and nine yeast species was constructed. Species-specific tRNA adaptation index (tAI), a measurement that evaluates translation efficiency of coding sequences [17], was predicted for each gene based on tRNA pool in that species. Because estimation of codon usage can be affected by species-specific factors such as genome-wide GC contents (Additional file 1), in order to have cross species comparisons, we normalized the tAI value of each gene by the median tAI value of all studied orthologous genes in the same species. The tAI values from nine yeast species were used to calculate the likelihood of observing the tAI value for A. gossypii gene in each orthologous gene group.

Based on statistical significance at the 95\% confidence level, we divided all orthologous genes into three categories. Group I: 333 genes showing significantly higher tAI values in A. gossypii than in yeasts (Figure 1A), group II: 552 genes showing significantly lower tAI values in A. gossypii than in yeasts (Figure $1 \mathrm{~B}$ ) and group III: the rest of 2,266 genes that have no obviously different tAI values between $A$. gossypii and yeasts (Additional file 2 for tAI values of genes in Group III). Under the assumption that translation efficiency is a selected trait, genes in group I would have higher expression and be translated more efficiently in A. gossypii than their orthologous genes in yeasts, whereas genes in group II would show the opposite pattern.

\section{GO (Gene Ontology) functional enrichment analysis}

Different tAI values for orthologous genes indicate the distinct functional importance of these genes in compared species [15]. Using GO terms in S. cerevisiae as reference, we characterized functional enrichment for Group I and Group II genes, respectively [18]. As shown in Figure 2A (See additional file 3 for the table list of these functional categories), Group I genes display significant enrichment in functions such as cell cycle regulation, mitosis and cytoskeleton organization. Interestingly, these functions have been hypothesized to be important for the unique life style in A. gossypii [6]. In addition, Group I genes also show enrichment of functions that are related with reproduction process, spore wall assembly and developmental process. By contrast, Group II genes show functional enrichment in amino acid and steroid biosynthesis (Figure $2 \mathrm{~B}$ ). Different from above two groups, Group III genes do not show any functional enrichment (data not shown).

\section{KEGG (Kyoto Encyclopedia of Genes and Genomes) pathway analysis}

In order to further see the impact of differences in gene translational efficiency, we assigned all studied orthologous genes into identified pathways according to KEGG annotation for S. cerevisiae [19]; 897 genes (75\% of all annotated genes in KEGG pathways) are included in our orthologous matrix. Among them, 79 genes belong to Group I and 219 genes belong to Group II. A Chi-square test was used to estimate distribution bias for each pathway. With a cut-off $P$ value of 0.01 , six pathways show significant distribution bias (Table 1). Consistent with gene ontology analysis, two of the three pathways enriched with Group I genes (cell cycle pathway and ubiquitin mediated proteolysis) are related to cell cycle regulation. 
A

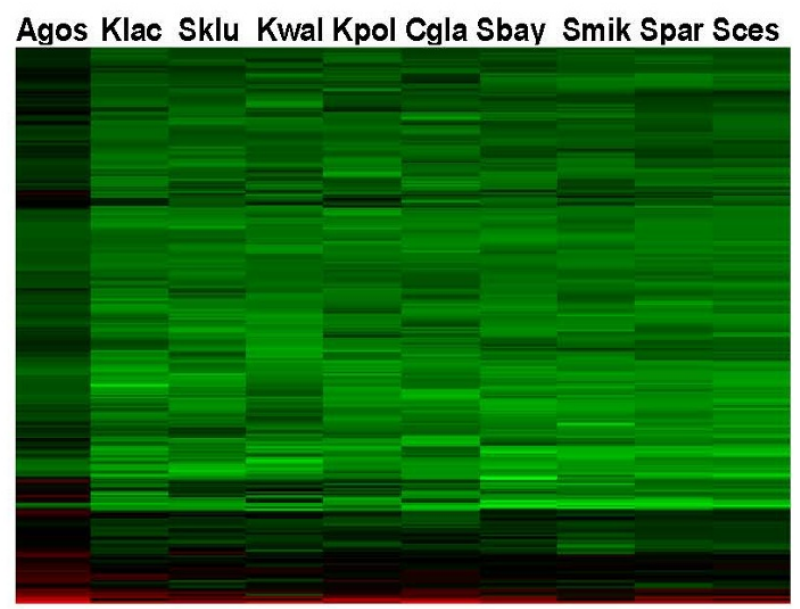

B

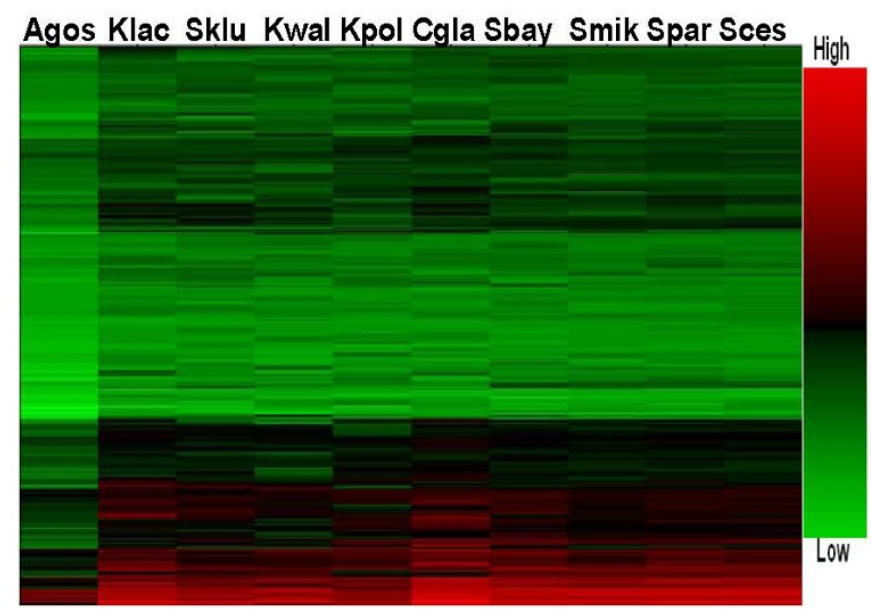

\section{Figure I}

tAI profile of orthologous gene in Group I and II. A) tAl values for orthologous genes in Group I (tAI values in A. gossypii are significantly higher than those in yeasts); B) tAl values for orthologous genes in Group II (tAl values in A. gossypii are significantly lower than those in yeasts). The scale of tAl values is shown to the right of the figure. (Agos: Ashbya gossypii; Sces: Saccharomyces cerevisiae; Spar:Saccharomyces paradoxus; Smik:Saccharomyces mikatae; Sbay:Saccharomyces bayanus; Cgla:Candida glabrata; Kpol:Kluyveromyces polysporus; Kwal:Kluyveromyces waltii, Sklu: Saccharomyces kluyveri; Klac:Kluyveromyces lactis).

As shown in Figure 3, cell cycle genes that belong to Group I scatter at each phase of cell division, so the enhanced translation efficiency, hence the increased importance of these genes, would influence the whole cell division process in A. gossypii. It is noteworthy that some cell cycle genes also belong to Group II. Interestingly, almost half of them are related with two protein complexes (condensin and mini-chromosome maintenance complex, Figure 3). Group II genes are enriched in metabolic processes such as lysine biosynthesis, steroid biosynthesis and aminoacyl-tRNA biosynthesis. At 95\% confidence level another six biosynthesis pathways are enriched with Group II genes (See additional file 4: Table 1 for the details of these pathways).

\section{Comparative analysis with experimentally confirmed genes in A. gossypii}

The above observations are all based on functional annotations for genes in $S$. cerevisiae. In order to confirm that genes with significantly higher tAI values in A. gossypii are truly enriched in cell cycle pathway genes, we identified all studied genes in A. gossypii according to NCBI database. Twenty one genes in A. gossypii were confirmed to be related with cell cycle processes (Table 2). Eight of them have significantly higher tAI values in A. gossypii (Group I, 333 gene), the other 13 genes do not show significant differences of tAI values among species (Group III, 2,266 genes). Fisher's exact test indicates that these confirmed cell cycle genes in A. gossypii are also enriched in Group I $(P=0.003)$.

\section{Discussion}

Codon usage bias in A. gossypii

Among studied species, A. gossypii has the highest genome-wide GC content (0.53), while the GC contents in other species are from 0.35 to 0.46 (Additional file $1 \mathrm{~A}$ ). Correlation coefficients of codon usage between A. gossypii and other nine species show strongly positive relationship with their GC contents, indicating that GC content might intensively shape codon usage in $A$. gossypii. Relationships of GC contents (estimated by GC3s: GC content at the third site of each codon) and codon usage bias (measured by Nc: effective number of codons which is a parameter measuring overall gene codon bias) on further analysis in all studies species show us that the higher GC3s is, the stronger correlation between GC3s and Nc is (Additional file 1B). For all studied orthologs in A. gossypii, GC3s is strongly correlated with $\mathrm{Nc}$, but not strongly associated with $\mathrm{tAI}$ and CAI (codon adaptive index) (Additional file 1C), indicating that change of GC content might have a larger impact on gene Nc than on tAI and CAI values. As in Man and Piple [15], we obtained low correlations between tAI and Nc, and between CAI and Nc (Additional file $1 \mathrm{C}$ ), which might be due to the dramatic change of GC 

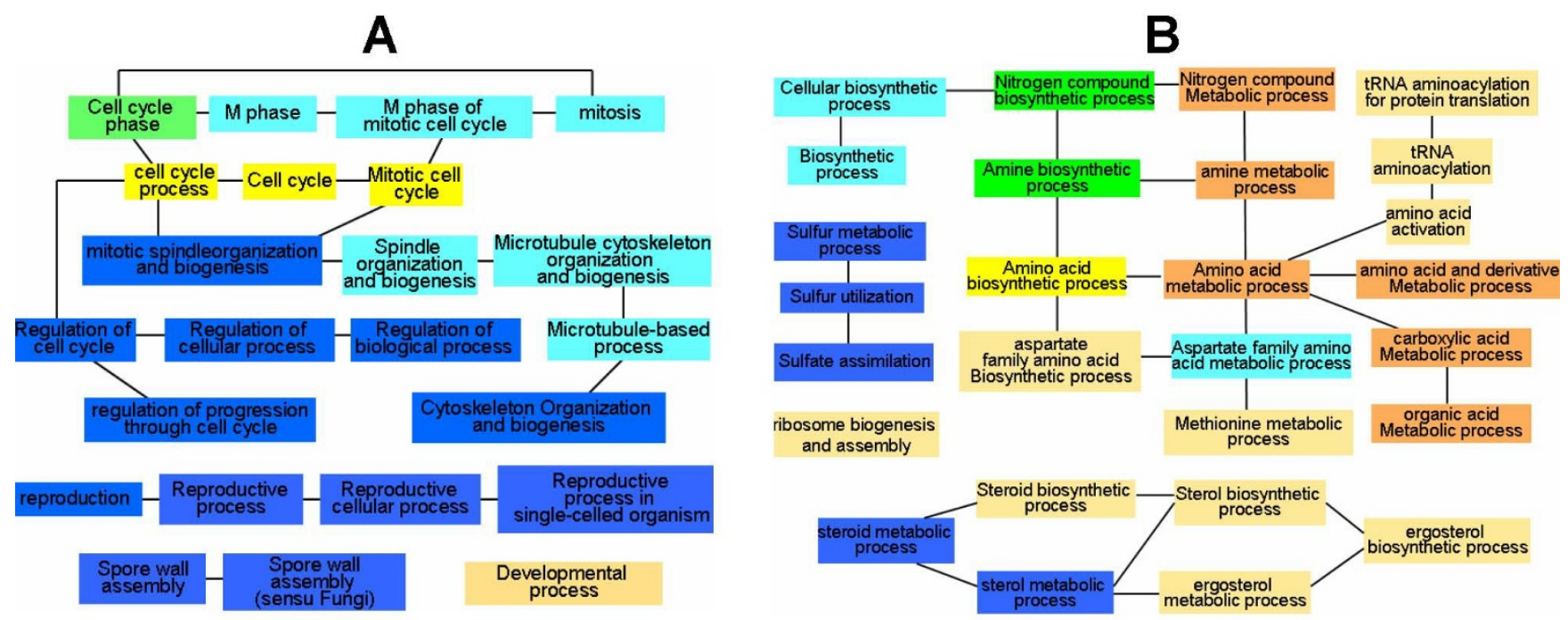

$<=1 \mathrm{e}-10$ $1 \mathrm{e}-10$ to $1 \mathrm{e}-8$

$1 e-8$ to $1 e-6$
$1 e-6$ to $1 \mathrm{e}-4$

$1 e-4$ to $1 e-2$

\section{Figure 2}

Distribution of enriched GO functions for genes in Group I and II. A) Biological functions with enriched gene representation in Group I; B) Biological functions with enriched gene representation in Group II. The edges between functional categories represent their hierarchical relationships in Gene Ontology annotation database. The colour of each box denotes $P$ value from GO term finder software (See additional file 3 for the table list of these functional categories with FDR analysis).

content, thus Nc values in A. gossypii. Nevertheless, both correlations are still statistically significant, while tAI and CAI show a very strong positive correlation in this species (Additional file 1C). Because tAI values for orthologs among studied species were normalized to remove the impact caused by some factors at genome level including GC content, it would still be an accurate indicator for expression level, thus the importance of A. gossypii genes during evolution.

\section{Possible effect of protein size on gene translation efficiency}

Natural selection biases synonymous codon usage to enhance accuracy and speed of protein synthesis in various organisms $[11,12]$. Differential gene translation efficiency reflects differences in functional requirements for proteins among organisms [14,15]. However, as previously reported, the negative correlation between translation efficiency and gene length indicates that translation efficiency could also be affected by protein size [14]. We

Table I: KEGG pathways that are enriched in Group I or Group II genes

\begin{tabular}{|c|c|c|c|c|c|c|}
\hline Pathway Name & KEGG ID & Total gene number & $\begin{array}{l}\text { Gene num. in } \\
\text { Group I }\end{array}$ & $\begin{array}{l}\text { Gene num. in } \\
\text { Group II }\end{array}$ & $\begin{array}{l}\text { Gene num. in } \\
\text { Group III }\end{array}$ & $P$-value \\
\hline Cell cycle - yeast a & $04 I I I$ & 83 & 19 & 12 & 52 & $<0.01$ \\
\hline $\begin{array}{l}\text { Basal transcription } \\
\text { factors a }\end{array}$ & 03022 & 23 & 6 & 1 & 16 & $<0.01$ \\
\hline $\begin{array}{l}\text { Ubiquitin mediated } \\
\text { proteolysis a }\end{array}$ & 04120 & 20 & 7 & 2 & 11 & $<0.01$ \\
\hline Lysine biosynthesis & 00300 & 14 & 0 & 10 & 4 & $<0.01$ \\
\hline $\begin{array}{l}\text { Biosynthesis of } \\
\text { steroids b }\end{array}$ & 00100 & 16 & 0 & 10 & 6 & $<0.01$ \\
\hline $\begin{array}{l}\text { Aminoacyl-tRNA } \\
\text { biosynthesis b }\end{array}$ & 00970 & 34 & 0 & 16 & 18 & $<0.01$ \\
\hline
\end{tabular}

a: Enriched with Group I genes; b: Enriched with Group II genes. 


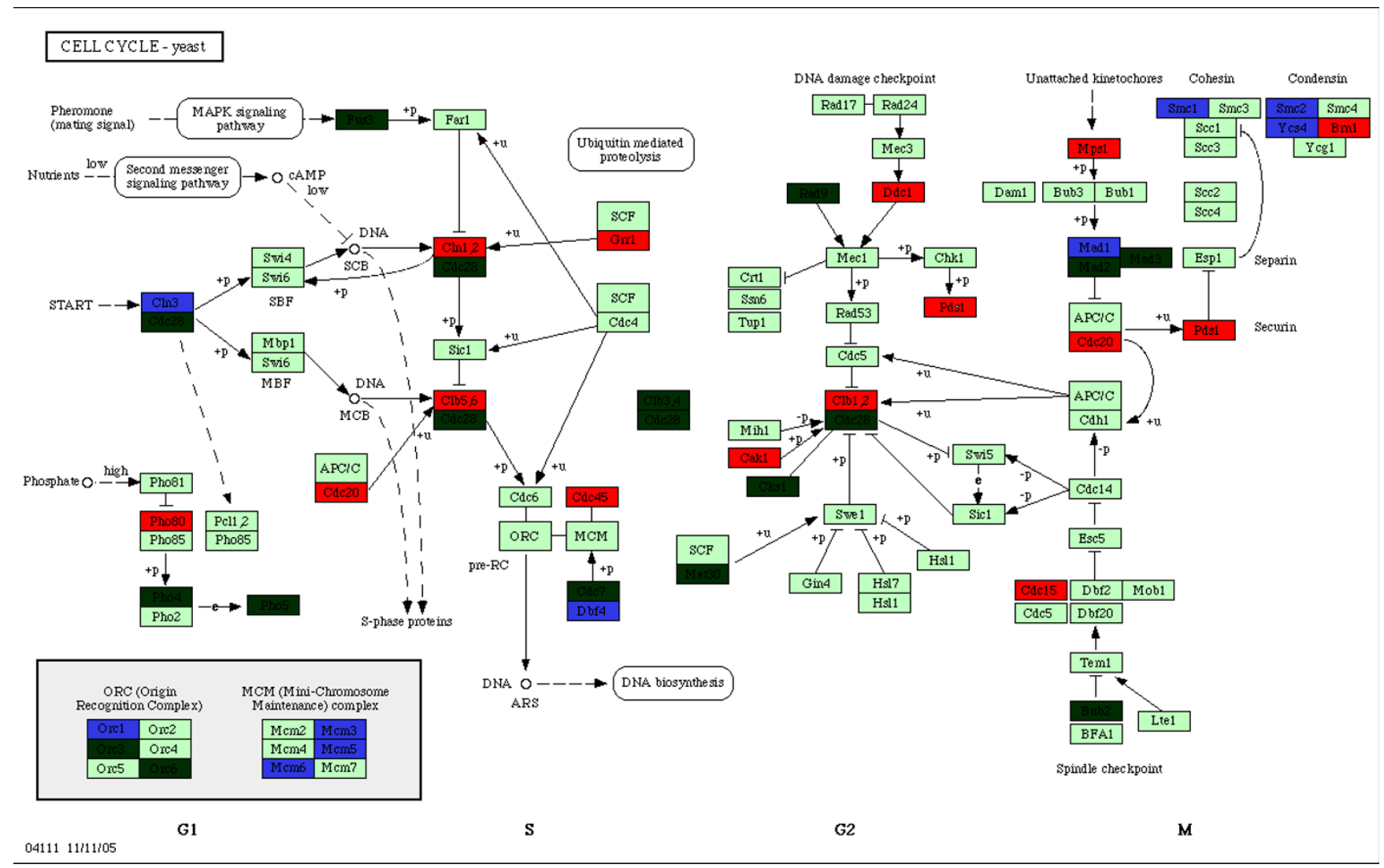

Figure 3

Cell cycle pathways in S. cerevisiae. The pathway figure was downloaded from KEGG database. Genes from Group I, II and III were denoted in red, blue and light green, respectively. Genes with dark green were not included in our orthologous matrix because they might be missing from one of the studied species.

tested if the different gene translation efficiencies observed between A. gossypii and yeast species could be caused by protein size deviation in different species. At first, we computed correlation coefficient of the tAI difference for each orthologs between A. gossypii and other nine yeasts and their protein size differences. To do this, for each orthologous gene, we calculated a protein size ratio, defined as the protein size of orthologous gene in A. gossypii (average protein size was used if two or more genes are in the same orthologous group) divided by the average protein size of orthologous genes in nine yeast species. Pearson correlation coefficient between tAI difference and protein size difference doesn't show significant association between the two $(r=0.035, P$-value $>0.05)$. In addition, Distributions of protein size ratio for genes in Group I and II are shown in Figure 4. There are no significant differences in protein size between A. gossypii and yeast spe- cies for both Group I and Group II genes, indicating that our observations are not the results of protein size change of orthologous genes during evolution.

\section{Higher translation efficiency of cell cycle genes in A. gossypii}

Comparison of translation efficiencies for orthologous genes should uncover regulation divergence, and thus the differential functional importance of those genes in diverged species $[14,15]$. Here by a comparative genomic approach, we identified two groups of orthologous genes showing differential translation efficiency between the filamentous fungus $A$. gossypii and its closely related yeast species. Genes in Group I have significantly higher translation efficiency in A. gossypii than those in yeasts. Functional analysis indicates that these genes are significantly enriched in cell cycle processes. Subsequent analysis of 
Table 2: Experimentally confirmed cell cycle genes in A. gossypii

\begin{tabular}{|c|c|c|c|}
\hline Gene name in $A$. gossypii & Orthologous ORF name in S. cerevisiae & $P$-value a & References \\
\hline $\mathrm{AgCDCl} 16$ & YKL022C & 0.000243 & Gladfelter et al. 2007 [35] \\
\hline $\mathrm{AgCDC} 42$ & YLR229C & 0.00143 & Wendland et al. 200I [36] \\
\hline $\mathrm{AgCDC} 24$ & YAL04IW & 0.001578 & Wendland et al. 200I [36] \\
\hline $\mathrm{AgCDC} 20$ & YGLII6W & 0.001869 & Gladfelter et al. 2007 [35] \\
\hline $\mathrm{AgDOCl}$ & YGL240W & 0.004167 & Gladfelter et al. 2007 [35] \\
\hline $\mathrm{AgRHO} 4$ & YKR055W & 0.004777 & Wendland et al. 2005 [4] \\
\hline AgCLB5 & YPRI20C & 0.007449 & Hungerbuehler et al. 2007 [37] \\
\hline AgPDSI & YDRII3C & 0.048015 & Gladfelter et al. 2007 [35] \\
\hline AgBEM2 & YERI55C & 0.07874 & Wendland 2000a [38] \\
\hline AgSWEI & YJLI87C & 0.117698 & Helfer et al. 2006 [39] \\
\hline AgBEM3 & YPLII5C & 0.122939 & Wendland et al. 2005 [4] \\
\hline $\mathrm{AgCDHI}$ & YGL003C & 0.127995 & Gladfelter et al. 2007 [35] \\
\hline AgRSRI & YGRI52C & 0.148734 & Bauer et al. 2004 [40] \\
\hline $\mathrm{AgCDC} 23$ & YHRI66C & 0.198647 & Gladfelter et al. 2007 [35] \\
\hline $\mathrm{AgBOII}$ & YBL085W & 0.2807 & Knechtle et al. 2006 [4I] \\
\hline $\mathrm{AgBNII}$ & YNL27IC & 0.281555 & Schmitz et al. 2005 [42] \\
\hline $\mathrm{AgSICI}$ & YLR079W & 0.787007 & Gladfelter et al. 2006 [20] \\
\hline AgRHOI & YPRI65W & 0.800873 & Walther et al. 2005 [43] \\
\hline AgSPA2 & YLL02IW & 0.812361 & Knechtle et al. 2003 [44] \\
\hline AgBUD3 & YCLOI4W & 0.821338 & Wendland 2003 [45] \\
\hline $\mathrm{AgCYKI}$ & YPL242C & 0.90517 & Wendland 2002 [46] \\
\hline
\end{tabular}

a: Comparison of tAl values between A. gossypii and other yeast species.

known pathways and experimentally studied genes in $A$. gossypii support such conclusion. Because enhanced translation efficiency indicates increased functional requirement for a gene, our results suggest that the roles of cell cycle genes might be substantially different between $A$. gossypii and yeasts. This suggestion is consistent with the observation that cell cycle processes, such as polar growth modes, cell cycle control and asynchronous nuclear division, are more complex in A. gossypii than those in yeast species $[4,6,20,21]$. Further experimental investigations for regulatory changes of cell cycle genes in A. gossypii could provide more insights into this conclusion.

For most pathogenic yeasts, transition from yeast form to filamentous form (pseudohyphal growth) is a key mark for their pathogenicity $[1,22]$. It has been reported that cell cycle genes play important roles in the morphological transition $[1,23]$. Micro-array experiment also shows that cell cycle genes are more up-regulated in S. cerevisiae filamentous form than are those in yeast form [1]. Our results indicate that cell cycle genes possess higher translational efficiency, and thus might be functionally more important, in filamentous fungus than those in yeast. Therefore, understanding species difference between filamentous fungus and yeasts could be helpful for illustrating mechanisms underlying filamentous growth of pathogenic yeasts.

Higher translation efficiency for metabolic genes in yeasts Genes in Group II have significantly lower translation efficiency in A. gossypii than those in yeasts. Functional enrichment analysis indicates that these genes are enriched for metabolic process, such as amino acid and steroid biosynthesis. This functional distribution might be associated with rapid cell growth in yeasts through fermentative life style. All yeast species studied can carry out fermentative growth under anaerobic or aerobic conditions, but A. gossypii can only conduct aerobic respiration [24]. The cell division rate is usually much faster for fermentative than for respiratory growth [25]. For eukaryotic organisms, cell size is an important control at check points for cell division $[26,27]$. In order to attain critical size, cells need to synthesize cellular components much more rapidly during fermentative than during respiratory growth. Therefore the metabolic processes should be in greater demand in yeasts than that in A. gossypii. The difference in metabolic rate could lead to differential translation efficiency for metabolic genes between A. gossypii and yeasts.

\section{Conclusion}

Comparative studies revealed that more than 95\% genes in multicellular organism A. gossypii share orthologs with unicellular organism S. cerevisiae [5]. It has been proposed that different growth modes between these species were controlled by a very similar gene set [4-6]. Our studies of orthologous genes among A. gossypii and several yeast species show higher translation efficiency of cell cycle genes in A. gossypii, which is consistent with more complicated cell cycle processes in this multicellular organism. On the other hand, our results are also consistent with the faster growth of unicellular yeasts, which leads to greater 


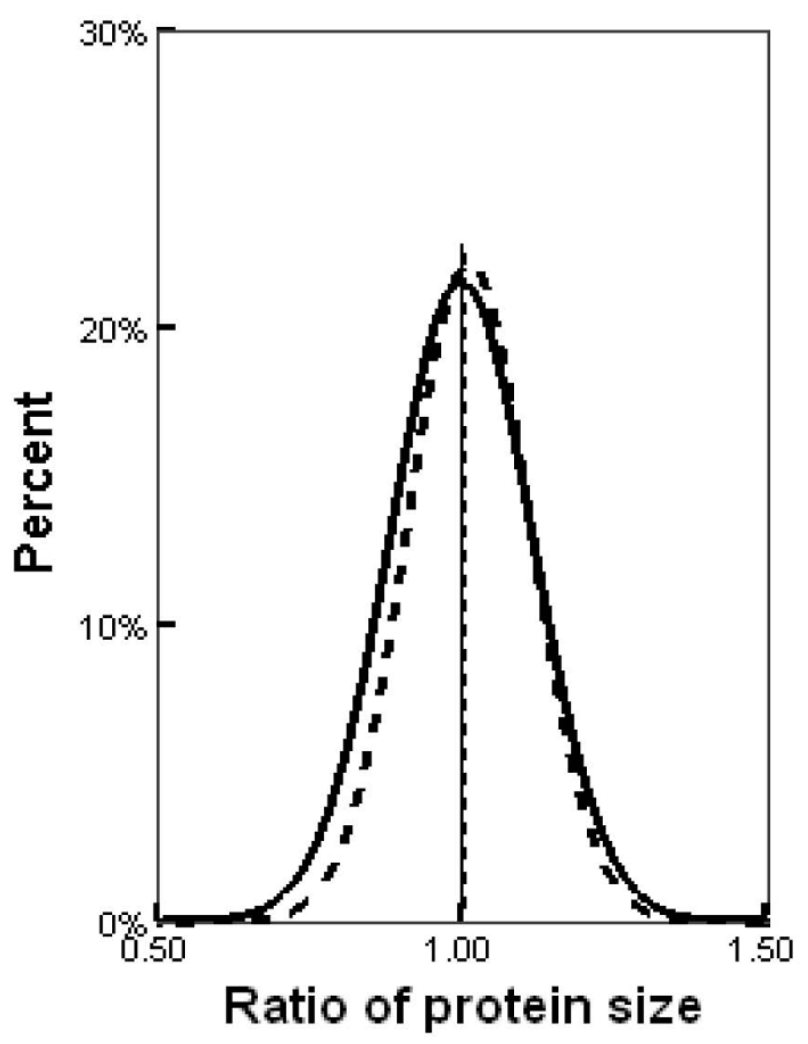

Figure 4

Protein size distribution for Group I and Group II genes. Protein size ratio for a gene was defined as the length of this gene in $A$. gossypii divided by the average length of its orthologous genes in nine yeast species. The solid and dashed lines represent the distributions of protein size ratio for Group I and Group II genes, respectively. Both distributions center around $I$, indicating that there are no protein length differences between $A$. gossypii and studied yeast species for both Group I and Group II genes.

demands of gene products for metabolic processes. Emergence of multicellular organisms was one of the most profound developmental transitions in the history of life [28]. Our results in this study, and further investigation for the difference between A. gossypii and yeast species might also be important for understanding this process.

\section{Methods}

\section{Species and sequence data}

According to the phylogenetic tree in Fitzpatrick et al. (2006) [2], 12 genomes have been sequenced in the clade Saccharomycotina. Among these species, only Ashbya gossypii is classified as filamentous fungus. For the remaining 11 yeast species, nine were used in our study (Saccharomyces cerevisiae, Saccharomyces paradoxus, Saccharomyces mikatae, Saccharomyces bayanus, Candida glabrata, Kluyveromyces polysporus, Kluyveromyces waltii, Saccharomyces kluyveri, and Kluyveromyces lactis). Saccharomyces kudriavzevii and Saccharomyces castellii were not used due to their low sequencing coverage. The protein, coding and genomic sequences for recently sequenced $K$. polysporus are from Scannell et al 2007 [29]. Sequences for other species were downloaded from Fungal Comparative Genomics database [30] and National Center for Biotechnology Information [31].

\section{Orthologous matrix}

Using InParanoid [32] program, orthologs between every two species were identified. According to the results from InParanoid, we used MultiParanoid [33] to generate orthologous matrix among all studied species. Each row in the matrix corresponds to an orthlogous gene shared by all studied species and each column to a species. Duplicated genes detected by InParanoid in one species were classified into the same orthologous group. Only orthologous groups which contain at least one gene for each species were used in our study. As a result, an orthologous matrix with 3,151 rows (genes) and 10 columns (species) was generated.

\section{tRNA adaptation index (tAI)}

First, we used tRNAscan-SE software (version 1.1) to predict copy numbers for all tRNA genes (tRNA gene pool) in each genome [34]. The tRNA pools among closely related species are conserved (See additional file 5 for the original data used to perform this analysis) [15]. For species with relatively low genome sequencing quality, we used tRNA pools from their closely related species, a same approach as in Ref 15: the predicted tRNA pool from $S$. cerevisiae was used to replace the ones in $S$. byanus, $S$. paradoxus, $S$. mikatae and the tRNA pool from $K$. lactis was used to replace the ones in K. waltii and S. kluyveri.

Second, given coding sequence for a gene, we used a modified tAI.R program [15] to estimate its tAI value in each species according to the tRNA pools (we kept all codons for methionines expect the start codons). When an orthologous group contains more than one gene in a species, the average tAI value of these genes was used for that species.

Finally, we normalized tAI value of each gene by median tAI value for all studied genes in the same species. For each orthologous group, we used the tAI values for nine yeast species to construct a normal distribution model, which was then used to estimate the probability of observing tAI value for the A. gossypii gene. Under 95\% confidence level, we identified 333 (Group I) and 552 genes (Group II) for which orthologs from $A$. gossypii have significantly higher and lower tAI values than those from yeasts, respectively. The rest 2,266 genes without significantly different tAI values between $A$. gossypii and yeasts were classified in Group III. 


\section{Functional enrichment analysis}

GO (Gene Ontology) annotations of S. cerevisiae genes were used for our analysis. We identified functional enrichment of genes in each group (group I, II and III) by GO term finder [18]. The functional enrichments were determined at $P$-value $<0.05$. Pathway information for $S$. cerevisiae was downloaded from KEGG database http:// www.genome.jp/kegg/. All studied orthologous genes in S. cerevisiae were mapped onto these pathways. For each pathway, a chi-square test was used to calculate the probability of gene number distribution among each group.

\section{Authors' contributions}

$\mathrm{HJ}$ and ZG designed the study. HJ and $\mathrm{YZ}$ analyzed the data. HJ, WW and ZG wrote the manuscript. JS prepared the figures and checked the manuscript. All authors read and approved the final manuscript.

\section{Additional material}

\section{Additional file 1}

GC content and codon usage in A. gossypii. Figure A shows the relationship between GC content and codon usage. $X$ axis is the GC content for each species. $Y$ axis is the correlation of whole genomic codon usage measured by RSCU (Relative Synonymous Codon Usage) between A. gossypii and other nine yeasts (Additional file 5). Figure B, using codonW http://codonw. sourceforge.net/, we calculated values for GC3s and $\mathrm{Nc}$ and obtained their correlation efficiencies in all studied species. $X$ axis is the GC contents for each species. $Y$ axis is the correlation coefficients between GC3s and Nc for each studied species. Figure C shows correlations among four indexes: tAI, GC3s, Nc and CAI in A. gossypii. All ribosomal protein gene sequences in $\mathrm{A}$. gossypii were used as reference set for CAI calculation. Pearson correlation coefficients among these parameters were calculated. The lower triangle of table contains the Pearson's correlation coefficients and the upper triangle of table contains P-values Click here for file

[http://www.biomedcentral.com/content/supplementary/14712148-8-343-S1.pdf]

\section{Additional file 2}

tAI values for orthologous genes in Group III. The figure shows the tAI values pattern for orthologous genes among all species in Group III (no significant tAI values between $\mathrm{A}$. gossypii and other yeast species). The scale of tAI values is shown to the right of the figure. (Agos: Ashbya gossypii; Sces: Saccharomyces cerevisiae; Spar:Saccharomyces paradoxus; Smik:Saccharomyces mikatae; Sbay:Saccharomyces bayanus; Cgla:Candida glabrata; Kpol:Kluyveromyces polysporus; Kwal:Kluyveromyces waltii, Sklu: Saccharomyces kluyveri; Klac:Kluyveromyces lactis)

Click here for file

[http://www.biomedcentral.com/content/supplementary/14712148-8-343-S2.pdf]

\section{Additional file 3}

The GO enrichment analyses for genes in Group I and II. This table presents all functional categories defined by GO finder with P-value $<$ 0.05 for genes in Group I and II.

Click here for file

[http://www.biomedcentral.com/content/supplementary/14712148-8-343-S3.xls]

\section{Additional file 4}

Additional KEGG pathways that show gene enrichment in Group I and II. This table presents all pathways with P-value between 0.05 and 0.01 .

Click here for file

[http://www.biomedcentral.com/content/supplementary/1471-

2148-8-343-S4.xls]

\section{Additional file 5}

The correlation of codon usage and tRNA pools among species. The lower triangle of the table contains the Spearman's correlation coefficients of codon usage in the whole genome. RSCU (Relative Synonymous Codon Usage) values were calculated for all codons in each species. Then correlations of RSCU values were calculated for each species pairs. The upper triangle of the table is the Spearman's correlation coefficients of tRNA gene number among all species.

Click here for file

[http://www.biomedcentral.com/content/supplementary/14712148-8-343-S5.xls]

\section{Acknowledgements}

We thank Chuan Gao, Orna Man for technical assistance, Lin Xu for discussion and David Pinney for reading the manuscript. This work was supported by a NSFC key grant (No. 30430400) and a 973 Program of China (No. 2007CB815703-5) to WW and startup funds from Cornell University awarded to ZG.

\section{References}

I. Prinz S, Avila-Campillo I, Aldridge C, Srinivasan A, Dimitrov K, Siegel AF, Galitski T: Control of yeast filamentous-form growth by modules in an integrated molecular network. Genome research 2004, 14(3):380-390.

2. Fitzpatrick DA, Logue ME, Stajich JE, Butler G: A fungal phylogeny based on $\mathbf{4 2}$ complete genomes derived from supertree and combined gene analysis. BMC evolutionary biology 2006, 6:99.

3. Mickelson MN: The metabolism of glucose by Ashbya gossypii. Journal of bacteriology 1950, 59(5):659-666.

4. Wendland J, Walther A: Ashbya gossypii: a model for fungal developmental biology. Nature reviews 2005, 3(5):42I-429.

5. Dietrich FS, Voegeli S, Brachat S, Lerch A, Gates K, Steiner S, Mohr C, Pohlmann R, Luedi P, Choi S, et al:: The Ashbya gossypii genome as a tool for mapping the ancient Saccharomyces cerevisiae genome. Science 2004, 304(5668):304-307.

6. Philippsen P, Kaufmann A, Schmitz HP: Homologues of yeast polarity genes control the development of multinucleated hyphae in Ashbya gossypii. Current opinion in microbiology 2005, 8(4):370-377.

7. Wolfe $\mathrm{KH}$ : Comparative genomics and genome evolution in yeasts. Philosophical transactions of the Royal Society of London 2006, 36I ( I 467):403-4I2.

8. McGregor AP, Orgogozo V, Delon I, Zanet J, Srinivasan DG, Payre F, Stern DL: Morphological evolution through multiple cis-regulatory mutations at a single gene. Nature 2007, 448(71 53): $587-590$.

9. Ikemura T: Correlation between the abundance of Escherichia coli transfer RNAs and the occurrence of the respective codons in its protein genes: a proposal for a synonymous codon choice that is optimal for the E. coli translational system. Journal of molecular biology I98I, I 5 I (3):389-409.

10. Ikemura $\mathrm{T}$ : Correlation between the abundance of yeast transfer RNAs and the occurrence of the respective codons in protein genes. Differences in synonymous codon choice patterns of yeast and Escherichia coli with reference to the abundance of isoaccepting transfer RNAs. Journal of molecular biology 1982, 158(4):573-597. 
1I. Sorensen MA, Kurland CG, Pedersen S: Codon usage determines translation rate in Escherichia coli. Journal of molecular biology 1989, 207(2):365-377.

12. Akashi H: Synonymous codon usage in Drosophila melanogaster: natural selection and translational accuracy. Genetics 1994, I 36(3):927-935.

13. Sharp PM, Li WH: The codon Adaptation Index - a measure of directional synonymous codon usage bias, and its potential applications. Nucleic acids research I987, I 5(3): | $28|-| 295$.

14. Akashi $\mathrm{H}$ : Translational selection and yeast proteome evolution. Genetics 2003, I 64(4): I29|-1303.

15. Man $O$, Pilpel $Y$ : Differential translation efficiency of orthologous genes is involved in phenotypic divergence of yeast species. Nature genetics 2007, 39(3):415-421.

16. Clarke B: Darwinian evolution of proteins. Science (New York, NY) 1970, I 68(934): I009-1011.

17. dos Reis M, Savva R, Wernisch L: Solving the riddle of codon usage preferences: a test for translational selection. Nucleic acids research 2004, 32( I 7):5036-5044.

18. Boyle El, Weng S, Gollub J, Jin H, Botstein D, Cherry JM, Sherlock G: GO::TermFinder - open source software for accessing Gene Ontology information and finding significantly enriched Gene Ontology terms associated with a list of genes. Bioinformatics (Oxford, England) 2004, 20( I 8):3710-37I5.

19. KEGG [http://www.genome.jp/kegg/]

20. Gladfelter AS, Hungerbuehler AK, Philippsen P: Asynchronous nuclear division cycles in multinucleated cells. The Journal of cell biology 2006, I 72(3):347-362.

21. Gladfelter AS: Nuclear anarchy: asynchronous mitosis in multinucleated fungal hyphae. Current opinion in microbiology 2006, 9(6):547-552.

22. Naglik J, Albrecht A, Bader O, Hube B: Candida albicans proteinases and host/pathogen interactions. Cellular microbiology 2004 6(10):915-926.

23. Rua D, Tobe BT, Kron SJ: Cell cycle control of yeast filamentous growth. Current opinion in microbiology 200I, 4(6):720-727.

24. Merico A, Sulo P, Piskur J, Compagno C: Fermentative lifestyle in yeasts belonging to the Saccharomyces complex. The FEBS journal 2007, 274(4):976-989.

25. Pfeiffer T, Schuster S, Bonhoeffer S: Cooperation and competition in the evolution of ATP-producing pathways. Science 200I, 292(55 I 6):504-507.

26. Calvert GR, Dawes IW: Cell size control of development in Saccharomyces cerevisiae. Nature 1984, 3 I 2(5989):6 I-63.

27. Rupes I: Checking cell size in yeast. Trends Genet 2002 I 8(9):479-485.

28. Maynard Smith J, Szathmary E: The Major Transitions in Evolution. UK 1995

29. Scannell DR, Frank AC, Conant GC, Byrne KP, Woolfit M, Wolfe KH: Independent sorting-out of thousands of duplicated gene pairs in two yeast species descended from a whole-genome duplication. Proceedings of the National Academy of Sciences of the United States of America 2007, 104(20):8397-8402.

30. Fungal Comparative Genomics database [http://fun gal.genome.duke.edu/]

31. The National Center for Biotechnology Information [http:// www.ncbi.nlm.nih.gov/]

32. Remm M, Storm CE, Sonnhammer EL: Automatic clustering of orthologs and in-paralogs from pairwise species comparisons. Journal of molecular biology 2001, 3 14(5): 1041-1052.

33. Alexeyenko A, Tamas I, Liu G, Sonnhammer EL: Automatic clustering of orthologs and inparalogs shared by multiple proteomes. Bioinformatics (Oxford, England) 2006, 22(I 4):e9- I5.

34. Lowe TM, Eddy SR: tRNAscan-SE: a program for improved detection of transfer RNA genes in genomic sequence. Nucleic acids research 1997, 25(5):955-964.

35. Gladfelter AS, Sustreanu N, Hungerbuehler AK, Voegeli S, Galati V, Philippsen P: The anaphase-promoting complex/cyclosome is required for anaphase progression in multinucleated Ashbya gossypii cells. Eukaryotic cell 2007, 6(2):182-197.

36. Wendland J, Philippsen P: Cell polarity and hyphal morphogenesis are controlled by multiple rho-protein modules in the filamentous ascomycete Ashbya gossypii. Genetics 200I, 157(2):601-610.
37. Hungerbuehler AK, Philippsen P, Gladfelter AS: Limited functional redundancy and oscillation of cyclins in multinucleated Ashbya gossypii fungal cells. Eukaryotic cell 2007, 6(3):473-486.

38. Wendland J, Philippsen P: Determination of cell polarity in germinated spores and hyphal tips of the filamentous ascomycete Ashbya gossypii requires a rhoGAP homolog. Journal of cell science 2000, I I3(Pt 9): |6 | |-1621.

39. Helfer H, Gladfelter AS: AgSwe I p regulates mitosis in response to morphogenesis and nutrients in multinucleated Ashbya gossypii cells. Molecular biology of the cell 2006, I 7( I 0):4494-45 I2.

40. Bauer Y, Knechtle P, Wendland J, Helfer H, Philippsen P: A Ras-like GTPase is involved in hyphal growth guidance in the filamentous fungus Ashbya gossypii. Molecular biology of the cell 2004, I 5( I 0):4622-4632.

4I. Knechtle P, Wendland J, Philippsen P: The SH3/PH domain protein AgBoil/2 collaborates with the Rho-type GTPase AgRho3 to prevent nonpolar growth at hyphal tips of Ashbya gossypii. Eukaryotic cell 2006, 5(10): 1635-1647.

42. Schmitz HP, Kaufmann A, Kohli M, Laissue PP, Philippsen P: From function to shape: a novel role of a formin in morphogenesis of the fungus Ashbya gossypii. Molecular biology of the cell 2006 , I7(I): I 30-I45.

43. Walther A, Wendland J: Initial molecular characterization of a novel Rho-type GTPase RhoH in the filamentous ascomycete Ashbya gossypii. Current genetics 2005, 48(4):247-255.

44. Knechtle $P$, Dietrich F, Philippsen P: Maximal polar growth potential depends on the polarisome component AgSpa2 in the filamentous fungus Ashbya gossypii. Molecular biology of the cell 2003, I4(10):4|40-4I54

45. Wendland J: Analysis of the landmark protein Bud3 of Ashbya gossypii reveals a novel role in septum construction. $E M B O$ reports 2003, 4(2):200-204.

46. Wendland J, Philippsen P: An IQGAP-related protein, encoded by $A g C Y K I$, is required for septation in the filamentous fungus Ashbya gossypii. Fungal Genet Biol 2002, 37(I):8I-88.
Publish with Biomed Central and every scientist can read your work free of charge

"BioMed Central will be the most significant development for disseminating the results of biomedical research in our lifetime. "

Sir Paul Nurse, Cancer Research UK

Your research papers will be:

- available free of charge to the entire biomedical community

- peer reviewed and published immediately upon acceptance

- cited in PubMed and archived on PubMed Central

- yours - you keep the copyright
BiolMedcentral 